\title{
Receptores MLSE/PSP Baseados em Algoritmos LMS de Passo Variável
}

\author{
Antonio Marques Arraes Filho, Ernesto Leite Pinto e Juraci Ferreira Galdino
}

\begin{abstract}
Resumo-Este artigo investiga o desempenho de receptores MLSE/PSP que usam algoritmos LMS de passo variável para estimação e rastreio de canais variantes no tempo. Alguns algoritmos LMS de passo variável são brevemente revistos, e se avalia a robustez de seu desempenho à escolha de parâmetros usados nas respectivas regras de adaptação do passo. Em seguida avalia-se o desempenho de receptores MSLE/PSP baseados nestes algoritmos através da simulação em computador, tendo como referências o algoritmo LMS convencional e o RLS. São avaliados diversos cenários de transmissão, em termos de razão sinal-ruído e de variabilidade do canal. Os resultados obtidos mostram que o esquema MLSE/PSP que emprega um algoritmo de passo variável recentemente proposto (MVSS-LMS) tem excelentes características de desempenho.
\end{abstract}

Palavras-Chave- receptores MLSE/PSP, processamento por percurso sobrevivente, algoritmo LMS de passo variável, canais duplamente seletivos, canais WSS-US, comunicações móveis

Abstract-The performance of MLSE/PSP receivers using variable step-size LMS algorithms to estimate and track time-varing channels is addressed in this paper. Some LMS algorithms are briefly reviewed and their robustness to the choice of parameters used in their rules of step-size adaptation is investigated. Several results of SER performance evaluation of MLSE/PSP receivers based on those algorithms are presented, taking the use of the conventional LMS and the RLS algorithm as references. Several transmission envrironments are considered in these evaluation, in terms of signal-to-noise ratio and channel variability. The results herein discussed show that the MLSE/PSP scheme based on one recently proposed variable step-size LMS algorithm (MVSSLMS) has very good performance characteristics.

Keywords-MLSE/PSP receivers, per-survivor processing, variable step-Size LMS algorithm, channel estimation, WSS-US channels, mobile communication, .

\section{INTRODUÇÃo}

A investigação de receptores adaptativos de elevado desempenho em canais duplamente seletivos tem sido um importante tema de pesquisa nos últimos anos. Um promissor campo de investigação neste contexto é o dos receptores de sequiências de símbolos baseados no critério de decisão de máxima verossimilhança que usam o princípio do processamento por percurso sobrevivente (MLSE/PSP, do inglês Maximum-Likelihood Sequence Estimation using Per-Survivor Processing) [1], [2], [3], [4]. O interesse por este tipo de receptor tem crescido bastante também devido à possibilidade de sua aplicação na decodificação de códigos espaço-temporais de treliça diante de desvanecimento plano variante no tempo [5], [6].

Antonio Marques Arraes Filho, Ernesto Leite Pinto e Juraci Ferreira Galdino, Departamento de Engenharia Eletrica, Instituto Militar de Engenharia, Rio de Janeiro, Brasil, E-mails: antonio.arraes@yahoo.com, ernesto@ime.eb.br, galdino@ime.eb.br.
De maneira geral os receptores MLSE/PSP têm características de desempenho muito superiores às de outros esquemas de recepção adaptativa, mas têm sido muitas vezes preteridos para aplicações práticas, em virtude de sua elevada complexidade de implementação. Assim sendo, a investigação de alternativas MLSE/PSP de complexidade reduzida é um tópico de grande interesse [7], [3], [4].

Como este tipo de receptor emprega um conjunto de estimadores de canal implementados através de filtros adaptativos, a sua complexidade computacional é fortemente afetada pela complexidade do algoritmo de filtragem usado [7], [3], [4], [6].

O emprego do algoritmo Least Mean Square (LMS) convencional tem sido considerado em alguns trabalhos, devido à sua baixa complexidade computacional. No entanto, o emprego de um valor de passo fixo limita as possibilidades de obtenção de melhores características de velocidade de convergência e de EMQ em regime permanente (rastreio) na estimação de canais variantes no tempo[3], [6].

Muitos trabalhos apresentados na literatura propõem algoritmos LMS de passo variável [8], [9], [10], [11], [12], visando diferentes aplicações. Tais propostas são potencialmente interessantes para aplicação em receptores MLSE-PSP, pela possibilidade de produzirem melhor desempenho do que o LMS convencional, com um aumento de complexidade relativamente pequeno. De maneira geral estes algoritmos LMS de passo variável se caracterizam pelo uso de regras de variação do passo que dependem de um ou mais parâmetros. Assim sendo, o desempenho por eles produzido pode vir a ser afetado significativamente pelo ajuste destes parâmetros.

Apesar da relevância e atualidade do tema, não é do conhecimento dos autores do presente artigo nenhum trabalho publicado que trate da avaliação e comparação dos ganhos de desempenho que se pode obter com receptores MLSE/PSP baseados em algoritmos LMS de passo variável.

$\mathrm{O}$ presente artigo procura contribuir para o preenchimento desta lacuna, tendo como foco a aplicação deste tipo de receptor em canais seletivos em freqüência e variantes no tempo. Além disso, neste artigo também se avalia a sensibilidade de desempenho de alguns algoritmos LMS de passo variável a variações nos parâmetros utilizados na adaptação de passo.

$\mathrm{O}$ restante deste texto é organizado da seguinte maneira: na Seção II discute-se de forma concisa os esquemas de recepção MLSE/PSP; na Seção III são revistos brevemente os algoritmos LMS de passo variável aqui empregados e são apresentados resultados de avaliação da robustez de seu desempenho à escolha de parâmetros por eles empregados; na Seção IV avalia-se através de simulação computacional o desempenho de esquemas MLSE/PSP baseados no uso dos 
algoritmos apresentados na Seção III e, por fim, na Seção V são sumarizadas as conclusões do trabalho.

\section{RECEPTORES MLSE/PSP}

O modelo de sistema de comunicações aqui adotado para investigação de receptores MLSE-PSP é apresentado na Figura 1. Supõe-se o uso de modulação em fase e quadratura, filtros de transmissão e recepção casados com resposta conjunta do tipo Nyquist, e um modelo de canal WSS-US (Wide Sense Stationary-Uncorrelated Scattering), com perfil de potência de retardos discreto e espectro Doppler de Jakes [13].

A fim de introduzir o problema central abordado neste artigo, admite-se inicialmente que o canal de comunicação é conhecido, invariante no tempo e modelado por um filtro de duração finita (FIR, Finite Impulse Response), com coeficientes espaçados a intervalo de símbolos, $T$. O sinal recebido no instante $k T$, é dado por:

$$
r_{k}=\boldsymbol{w}^{H} \boldsymbol{s}_{k}+v_{k}
$$

onde $\boldsymbol{w}=\left[w_{0} \cdots w_{L-1}\right]^{T}$ é a resposta ao impulso (RI) do canal, $L$ é a quantidade de coeficientes dessa RI, $\boldsymbol{s}_{k}=$ $\left[s_{k} s_{k-1} \cdots s_{k-L+1}\right]$ é o vetor formado pelos símbolos que afetam $r_{k}$, e $v_{k}$ é um ruído branco gaussiano, de média nula e variância $\sigma_{v}^{2}$,

Neste caso, a sequiência MLSE pode ser expressa por:

$$
\boldsymbol{s}^{*}=\arg \min _{\boldsymbol{s}_{i}}\left\|\boldsymbol{r}-\boldsymbol{w}^{H} \boldsymbol{s}_{\boldsymbol{i}}\right\|^{2}
$$

e pode ser obtida com o algoritmo de Viterbi (AV) [14].

Quando a RI do canal é desconhecida e variante no tempo, a aplicação do critério MLSE leva à necessidade de uma busca conjunta de sequiências de estimativas da RI do canal e de símbolos, o que em geral é computacionalmente inviável.

Uma alternativa para contornar esta dificuldade poderia ser o uso de sequiências de treinamento para obter uma estimativas da RI do canal, $\hat{\boldsymbol{w}}$, a serem usadas na recepção dos dados. A regra de decisão (sub-ótima) ficaria dada por:

$$
\boldsymbol{s}^{*}=\arg \min _{\boldsymbol{s}_{i}}\left\|\boldsymbol{r}-\hat{\boldsymbol{w}}^{H} \boldsymbol{s}_{\boldsymbol{i}}\right\|^{2},
$$

Se o canal não puder ser considerado invariante ao longo do bloco de dados, ou seja, entre dois períodos de treinamento sucessivos, o receptor precisa rastrear a sua RI enquanto recebe os dados. A técnica mais empregada para isto consiste em utilizar os símbolos detectados como entrada para um algoritmo de filtragem adaptativa encarregado do rastreamento. Essa técnica, também empregada em outros tipos de equalizadores, é aqui denominada adaptação direcionada pela decisão (DD, do termo em inglês Decision-Directed).

A eficácia da DD em receptores adaptativos de seqüência de símbolos é, no entanto, bastante limitada, em razão dos atrasos de decisão. Uma alternativa eficaz para superar esta limitação em receptores MLSE é o emprego do princípio do processamento por percurso sobrevivente (PSP, do inglês PerSurvivor Processing) [1], [2].

Como ilustrado na Figura 1, um receptor MLSE/PSP utiliza um algoritmo de busca (seleção de seqüências sobreviventes) e um conjunto de filtros adaptativos. Estes filtros são encarregados de obter estimativas da RI do canal condicionadas às sequiências sobreviventes. Tais estimativas, por sua vez,

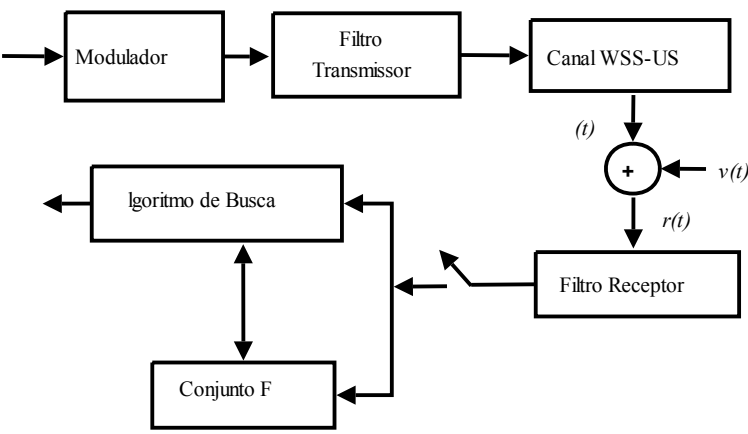

Fig. 1. Modelo de sistema de comunicações com receptor MLSE/PSP.

são empregadas para cálculo dos incrementos nas métrica de decisão associadas às extensões destas sequiências. As métricas acumuladas são em seguida utilizadas pelo algoritmo de busca para seleção de novas seqüências sobreviventes (de comprimento maior), e assim por diante.

As características de desempenho e complexidade computacional de um receptor MLSE/PSP dependem fundamentalmente das escolhas do algoritmo de busca e do algoritmo de filtragem adaptativa. Vários algoritmos de busca têm sido considerados, dentre os quais se destacam o algoritmo de Viterbi e o algoritmo M [15], [16]. No que se refere ao algoritmo de filtragem adaptativa, diversos trabalhos apontam as limitações do algoritmo LMS convencional [3], e a necessidade outras alternativas de baixa complexidade computacional com níveis de desempenho mais próximos dos obtidos com filtragem de Kalman e com o algoritmo RLS [7].

Neste trabalho usou-se o algoritmo de Viterbi para realizar a seleção das sequiências sobreviventes e, como algoritmo de filtragem adaptativa, investigou-se o uso de alguns algoritmos LMS de passo variável [12], [11], [9], tendo como referências os algoritmos RLS e LMS convencional.

Para obtenção de todos os resultados de simulação aqui reportados adotou-se um modelo WSS-US com três raios espaçados a intervalos de símbolos $(L=3)$. Diversos cenários de transmissão são considerados, em termos da razão sinalruído na entrada do receptor (RSR) e da variabilidade do canal, especificada pelo desvio Doppler máximo normalizado $\left(f_{D} T\right)$.

\section{Algoritmos LMS DE PASSo VARiável}

A regra de operação básica de um algoritmo LMS de passo variável é dada por $\hat{\boldsymbol{w}}(n+1)=\hat{\boldsymbol{w}}(n)+\mu(n) \boldsymbol{s}(n) \varepsilon^{*}(n)$ onde $\hat{\boldsymbol{w}}(n)$ é o vetor que contém as estimativas dos parâmetros de interesse no instante $n, \mu(n)$ denota o passo de adaptação usado no instante $n$, e $\varepsilon(n)$ é o erro entre a saída do filtro adaptativo $y(n)=\hat{\boldsymbol{w}}^{H}(n) \boldsymbol{s}(n)$ e o sinal desejado $d(n)$.

Apresenta-se a seguir os algoritmos LMS de passo variável empregados neste trabalho. São também discutidos alguns resultados de avaliação do desempenho destes algoritmos na estimação de canais. Como medida de desempenho usou-se o erro médio quadrático na estimação dos parâmetros (MSWE, da nomenclatura em Inglês Mean Square Weight Error).

Este parâmetro, aqui denotado por $D(n)$, é definido como o valor esperado do quadrado da norma do vetor erro de estimação dos coeficientes, ou seja, $D(n) \triangleq \mathrm{E}\{\| \boldsymbol{w}(n)-$ $\left.\hat{\boldsymbol{w}}(n) \|^{2}\right\}$. Cabe notar que o erro de estimação do sinal 


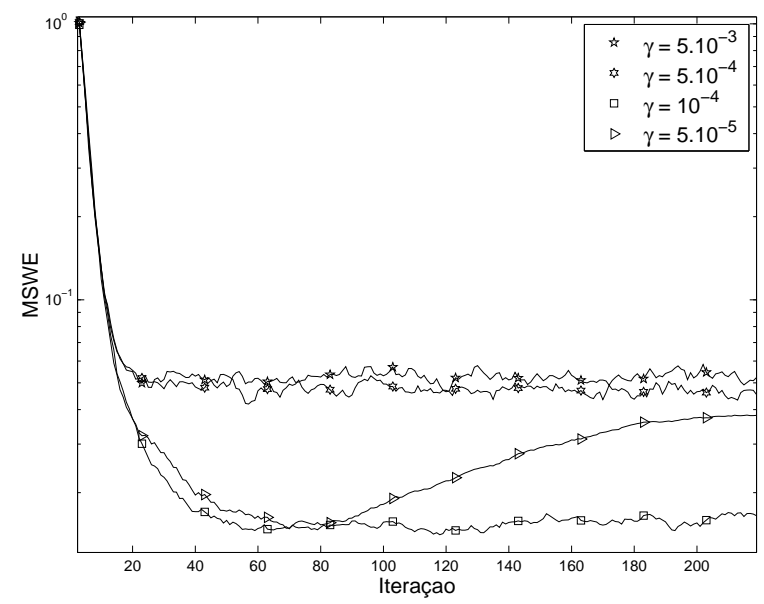

Fig. 2. Curvas de MSWE do algoritmo VSS-LMS obtidas com $f_{D} T=10^{-3}$ e $\mathrm{RSR}=10 \mathrm{~dB}$, para diferentes valores de $\gamma$.

desejado (MSE) pode ser obtido de forma imediata a partir do MSWE, da variância dos símbolos transmitidos e da variância do ruído. Optou-se neste trabalho pelo emprego do MSWE por ser ele um indicador mais direto da qualidade do estimador de canal.

Os resultados foram obtidos com modulação QAM-16, $f_{D} T=10^{-3}$ e RSR de $10 \mathrm{~dB}$. O MSWE foi estimado a partir de 1000 simulações independentes da transmissão de blocos de 250 símbolos. Outras condições de $f_{D} T$ e RSR, não incluídas neste artigo, mostraram características semelhantes às reportadas em seguida [17].

No que diz respeito especificamente ao produto $f_{D} T$, optouse por não empregar aqui valores acima de $10^{-3}$ porque estes são de menor interesse para aplicações práticas em sistemas de comunicações móveis e também porque se verificou em um trabalho anterior [2] que o desempenho de receptores MLSE/PSP baseados no algoritmo LMS convencional fica bastante degradado nestas condições.

\section{A. Algoritmo VSS-LMS}

Neste algoritmo a atualização do passo é controlada pela predição do erro, $\varepsilon(n)$, permitindo que o filtro adaptativo rastreie as variações do ambiente com o tempo e também produza um baixo EMQ em regime permanente. Este esquema foi denominado pelos seus autores de algoritmo VSS-LMS, de Variable Step-Size LMS [9].

O valor do passo é adaptado recursivamente da seguinte forma:

$$
\mu(n+1)=\alpha \mu(n)+\gamma \varepsilon(n)^{2}
$$

$\operatorname{com} 0<\alpha<1, \gamma>0$. Se $\mu(n+1)>\mu_{\max }$ então $\mu(n+1)=$ $\mu_{\max } ;$ se $\mu(n+1)<\mu_{\min }$ então $\mu(n+1)=\mu_{\text {min }}$.

$\mathrm{O}$ passo inicial $\mu(0)$ é geralmente estabelecido no valor de $\mu_{\max }$. Uma condição suficiente para garantir a convergência do algoritmo [18] é $\mu_{\max } \leq \frac{2}{3 \operatorname{tr}\left[\boldsymbol{R}_{s}\right]}$, sendo $\boldsymbol{R}_{s}$ a matriz de correlação da entrada do filtro adaptativo.

A Figura 2 ilustra as curvas de MSWE do algoritmo VSSLMS obtidas com diferentes valores de $\gamma$, mantendo-se $\alpha=$ 0.97. O valor de $\alpha$ foi fixado com base numa investigação prévia. Observa-se que o melhor desempenho do algoritmo

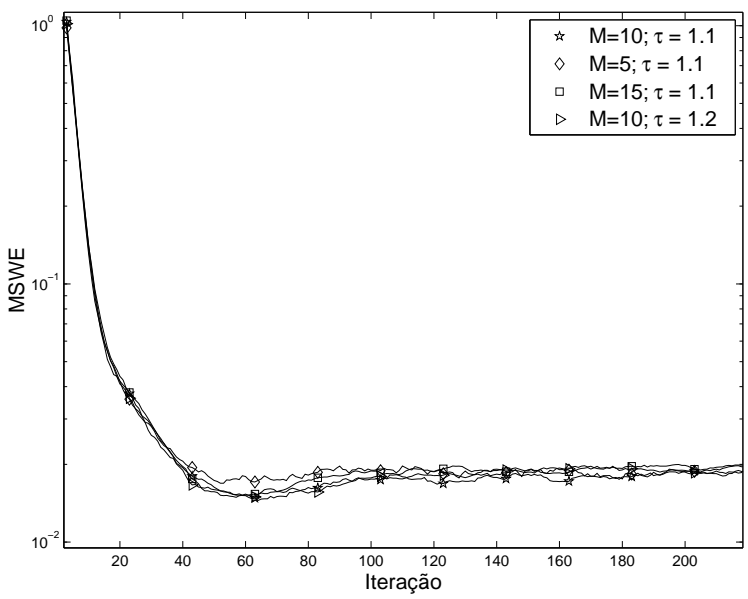

Fig. 3. Curvas de MSWE do algoritmo OVS-LMS variando-se os parâmetros $M$ e $\tau$, para $f_{D} T=10^{-3}$ e RSR $=10 \mathrm{~dB}$.

foi obtido com $\gamma=10^{-4}$, e que o uso de um valor um pouco diferente deste já degrada sensivelmente o seu desempenho.

\section{B. Algoritmo OVS-LMS}

Em [11] é proposto um algoritmo LMS de passo variável para ambiente não estacionário, tendo por base um trabalho anterior dos mesmos autores, voltado para a otimização do passo do algoritmo LMS em ambiente estacionário. Este algoritmo é aqui denominado OVS-LMS, do Inglês Optimum Variable step-size LMS.

A idéia chave do OVSS-LMS é realizar um processamento por blocos de amostras de comprimento $M$, nos quais o ambiente possa ser considerado aproximadamente invariante. Para cada bloco é usado um valor de passo fixo otimizado com base no trabalho acima mencionado, e também é obtida uma estimativa local do EMQ. Ao final de cada bloco esta estimativa é comparada com um limiar $\tau$, a fim de decidir sobre o valor de passo a ser utilizado no próximo bloco.

A Figura 3 apresenta as curvas de MSWE obtidas num dos experimentos realizados para avaliar a sensibilidade do seu desempenho à escolha dos parâmetros $M$ e $\tau$. Estas curvas são bem parecidas, e os valores dos parâmetros $M$ e $\tau$ que produziram um melhor compromisso entre velocidade de convergência e MSWE em regime permanente foram 10 e 1.1 , respectivamente.

\section{Algoritmo MVSS-LMS}

Em [12] foi apresentado um algoritmo de passo variável para estimação de canais WSS-US, obtido analiticamente sob o critério de minimização do MSWE. Em [19] obteve-se a seguinte equação de recursão para o passo variável ótimo:

$$
\mu_{\mathrm{opt}}(n+1)=\frac{\mu_{\mathrm{opt}}(n)-\mu_{\mathrm{opt}}(n)^{2} \sigma_{s}^{2}}{1-\mu_{\mathrm{opt}}(n)^{2} L \sigma_{s}^{4}}
$$

onde $\sigma_{s}^{2}$ é a variância dos símbolos de entrada e $L$ é o número de coeficientes da RI do canal. Esta recursão se inicia com $\mu_{\text {opt }}(1)=\sigma_{w}^{2} /\left[L\left(\sigma_{w}^{2} \sigma_{s}^{2}+\sigma_{v}^{2}\right)\right]$, sendo $\sigma_{\boldsymbol{w}}^{2}$ a soma das variâncias dos coeficientes do canal, e $\sigma_{v}^{2}$ a variância do ruído. 

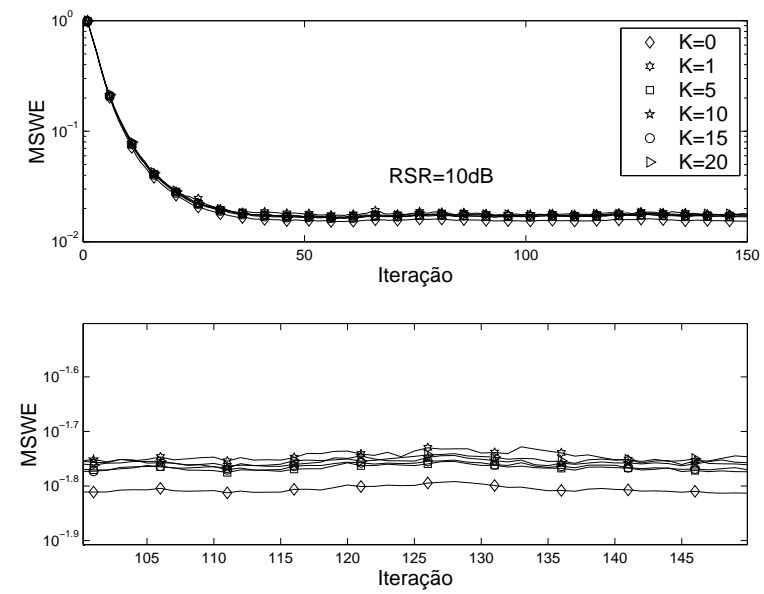

Fig. 4. Curvas de MSWE do algoritmo MVSS-LMS com erro na estimação da variância do ruído, para $f_{D} T=10^{-3}$ e RSR=10dB.

Nesta mesma referência se propõe uma regra para interromper a adaptação do passo, de modo a combinar as propriedades de aceleração da convergência e de minimização do MSWE em regime permanente, utilizando-se resultados da análise apresentada em [20]. O algoritmo assim obtido é chamado de MVSS-LMS, (de Minimum MSWE).

Mostra-se ainda em [19] que o algoritmo MVSS-LMS também é aplicável a canais invariantes no tempo, bastando para isso fixar a priori o nível de MSWE a ser atingido a partir da interrupção da adaptação do passo.

Para avaliar a robustez do algoritmo MVSS-LMS a erros na estimação da variância do ruído $\sigma_{v}^{2}$ admitiu-se o emprego de um estimador modelado da seguinte maneira:

$$
\hat{\sigma_{v}^{2}}=\left|\sigma_{v}^{2}+e_{\sigma_{v}^{2}}\right|
$$

onde $e_{\sigma_{v}^{2}}$ é uma variável aleatória gaussiana de média nula e variância $\sigma_{v_{\text {est }}}^{2}$. Note-se que se trata de um estimador polarizado, cuja polarização e variância aumentam com o aumento de $\sigma_{v_{e s t}}^{2}$.

A fim de estabelecer uma vinculação entre o valor de $\sigma_{v_{e s t}}^{2}$ e a RSR na entrada do receptor, utilizou-se a relação $\sigma_{\text {est }}^{2}=K\left(\sigma_{v}^{2} / \sigma_{s}^{2}\right)$, sendo o parâmetro $K$ usado para modelar diferentes níveis de qualidade do estimador.

Para levantamento das estimativas de MSWE foram realizadas simulações idênticas às anteriores, exceto pelo fato de que o valor de $\sigma_{v}^{2}$ utilizado pelo algoritmo MVSS-LMS foi obtido por sorteio, usando-se o modelo acima. Para cada bloco de amostras do sinal recebido foi realizado um sorteio independente. A Figura 4 apresenta alguns dos resultados obtidos com diversos valores do parâmetro $K$.

Pode ser visto que as curvas de MSWE produzidas com erros na estimação de $\sigma_{v}^{2}$ não se modificam significativamente com o aumento de $K$. Mesmo para uma alta polarização e variância do estimador $(K=20)$, os resultados foram bastante parecidos com os do caso em que se admite estimação perfeita $(K=0)$. Esses resultados indicam que o MVSSLMS de passo variável apresenta pouca sensibilidade a erros de estimação da variância do ruído. A mesma constatação foi feita com outros valores de RSR e de $f_{D} T$ [17].

Além disso, também foi feita uma investigação da sensibilidade de desempenho deste algoritmo a erros na estimação do

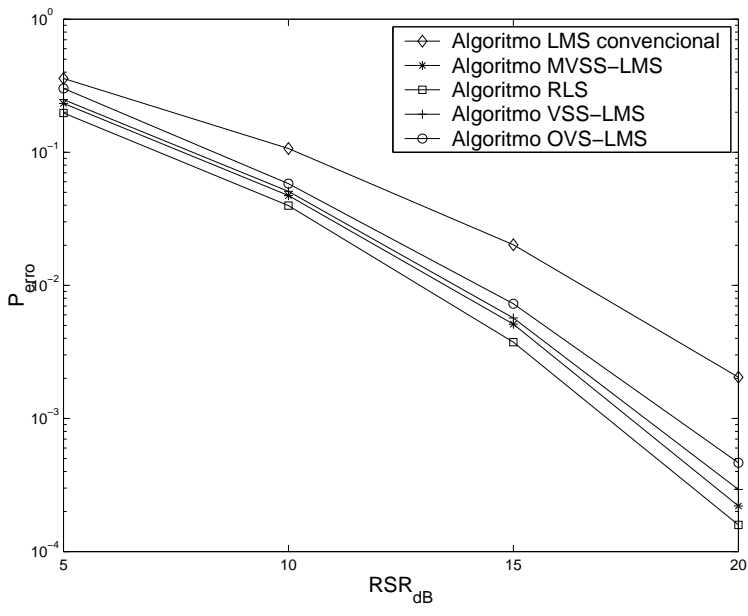

Fig. 5. Taxa de erro de símbolo de receptores MLSE/PSP baseados nos algoritmos investigados, com $f_{D} T=10^{-4}$.

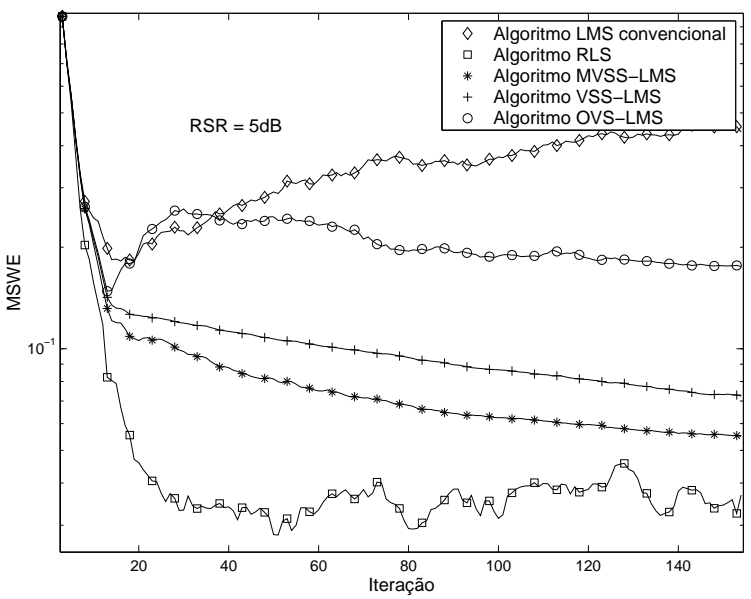

Fig. 6. Curvas de MSWE em receptores MLSE/PSP baseados nos algoritmos investigados, para $f_{D} T=10^{-4}$ e RSR $=5 \mathrm{~dB}$.

desvio Doppler máximo $f_{D}$, a qual mostrou ser o MVSS-LMS também bastante robusto a erros desta natureza [17].

\section{DESEMPENHO EM RECEPTORES MLSE/PSP}

Considerou-se neste caso a transmissão de blocos de 150 símbolos de uma constelação QPSK, sendo os dez primeiros símbolos utilizados como seqüência de treinamento. Assim, tem-se uma vazão de informação de $92,11 \%$. Foram simulados valores de RSR entre 5 e $20 \mathrm{~dB}$. O número de blocos transmitidos foi variado de acordo com a RSR adotada, a fim de se obter estimativas de taxas de erro confiáveis em toda faixa de RSR considerada.

Duas situações foram investigadas nas simulações. Na primeira delas, o desvio Doppler máximo normalizado foi fixado em $f_{D} T=10^{-4}$ e os parâmetros utilizados pelos algoritmos [8], [9], [11] foram ajustados para garantir o seu melhor desempenho. Na segunda, o produto $f_{D} T$ é modelado por uma variável aleatória uniforme no intervalo $\left[10^{-4}, 10^{-3}\right]$. No início de cada bloco o valor de $f_{D}$ é sorteado e mantido constante durante todo o bloco. Neste caso, os parâmetros dos algoritmos [9], [11] foram ajustados para $f_{D} T=10^{-4}$. 


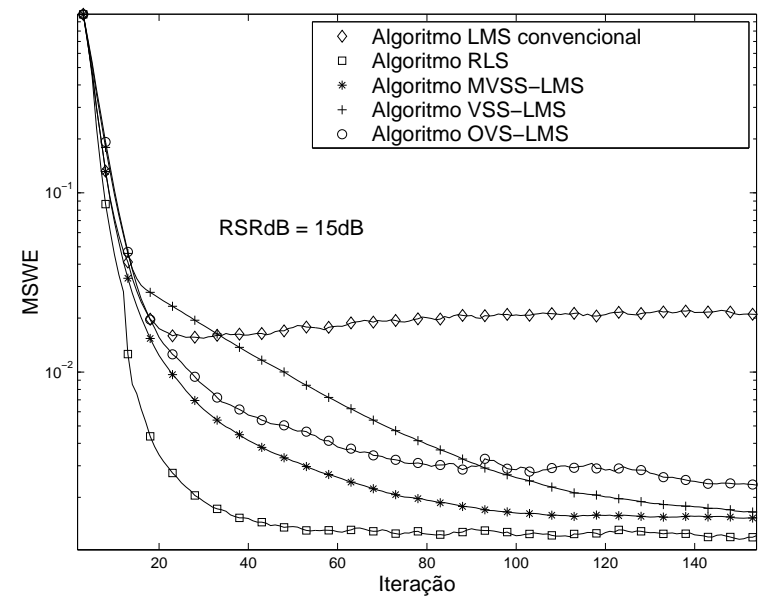

Fig. 7. Curvas de MSWE em receptores MLSE/PSP baseados nos algoritmos investigados, para $f_{D} T=10^{-4}$ e RSR $=15 \mathrm{~dB}$.

Para buscar um melhor desempenho do algoritmo LMS convencional utilizou-se dois valores de passo, um (maior) para o período de treinamento e outro para a recepção dos dados. O primeiro valor foi escolhido como sendo o passo inicial do algoritmo MVSS-LMS ( $\left.\mu_{\text {opt }}(1)\right)$, e o segundo foi feito igual a $\mu_{\text {opt }}(11)$, que é o valor de passo utilizado pelo algoritmo MVSS-LMS no início da recepção de dados.

A Figura 5 apresenta as curvas de taxa de erro de símbolo $\mathrm{X}$ RSR obtidas com os 5 algoritmos empregados para estimação de canal nos receptores MLSE-PSP, para $f_{D} T=10^{-4}$. Notase que o esquema baseado no algoritmo RLS foi o que apresentou a menor taxa de erro, seguido pelo que usa o algoritmo MVSS-LMS. Entre os esquemas baseados em LMS de passo variável, o que produziu piores resultados foi o que usa o algoritmo OVS-LMS. No entanto, mesmo este esquema ainda superou em desempenho o receptor baseado o LMS convencional.

A Figura 6 ilustra curvas de MSWE referentes às estimativas de canal obtidas com a seqüência de treinamento seguida da seqüência de dados selecionada por cada esquema MLSE-PSP (sequiência de melhor métrica). Estas curvas foram obtidas com RSR de 5dB, sob as mesmas condições de simulação empregadas na obtenção da Figura 5, e mostram que o emprego do LMS convencional e do OVS-LMS leva à ocorrência de valores elevados de MSWE no final do bloco de treinamento.

A Figura 6 mostra ainda que os demais algoritmos produziram rápida velocidade de convergência, especialmente o RLS, que atingiu o menor nível de MSWE em regime permanente, o que justifica a menor taxa de erro do receptor nele baseado. Dentre os algoritmos LMS de passo variável, o MVSS-LMS propiciou o melhor desempenho.

A Figura 7 ilustra as curvas de MSWE produzidas pelos algoritmos investigados para RSR $=15 \mathrm{~dB}$. Nela se observa que o algoritmo RLS novamente apresentou os melhores resultados, enquanto o algoritmo de passo variável com melhor desempenho (maior velocidade de convergência e menor nível de MSWE em regime permanente) foi o MVSS-LMS. Por outro lado, o algoritmo LMS de passo variável que apresentou o pior desempenho foi novamente o OVS-LMS.

Foram também obtidas curvas de MSWE em condições

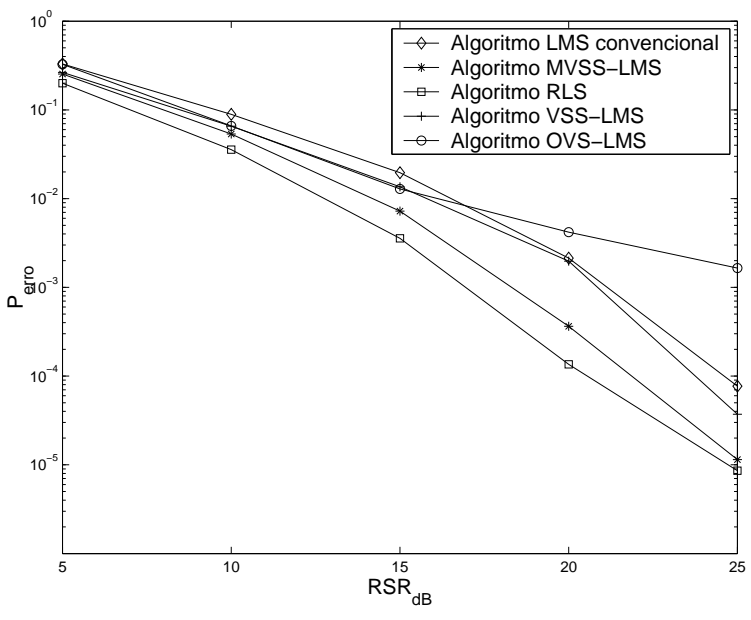

Fig. 8. Taxa de erro por símbolo utilizando receptores MLSE/PSP, para $f_{D} T$ aleatório.

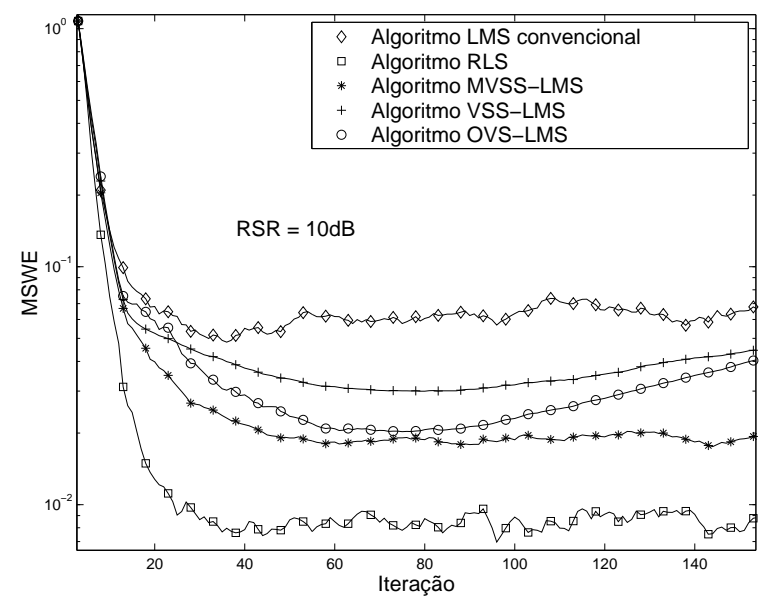

Fig. 9. Curvas de MSWE em receptores MLSE/PSP baseados nos algoritmos investigados, com $f_{D} T$ aleatório e $\mathrm{RSR}=10 \mathrm{~dB}$.

idênticas às anteriores, a menos da RSR, que foi fixada em $20 \mathrm{~dB}$. Verificou-se que o esquema com MVSS-LMS novamente apresentou o melhor desempenho de MSWE entre os receptores baseados em algoritmos LMS de passo variável.

A Figura 9 apresenta as curvas de taxa de erro de símbolo $X$ RSR obtidas modelando-se o produto $f_{D} T$ como uma variável aleatória. Nela pode-se ver que, para todos os valores de RSR testados, o receptor MLSE-PSP baseado no algoritmo RLS obteve a menor taxa de erro de símbolos, seguido pelo esquema que usa o algoritmo MVSS-LMS. Observa-se também que à medida que se aumenta o valor da RSR os ganhos de desempenho produzidos por estes dois receptores ficam mais evidentes, e que para RSR $=25 \mathrm{~dB}$ ambos apresentam valores de taxa de erro bastante próximos.

A Figura 10 ilustra as curvas de MSWE obtidas com a mesma modelagem do produto $f_{D} T$ como variável aleatória e com RSR $=10 \mathrm{~dB}$. Vê-se claramente que o esquema baseado no algoritmo RLS apresenta alta velocidade de convergência, atingindo valores de MSWE em regime permanente da ordem de $9,3 \times 10^{-3}$. A taxa de erro de símbolo deste receptor foi de 0,035 , aproximadamente. O esquema que usa o algoritmo MVSS-LMS atingiu um nível de MSWE em regime perma- 
nente em torno de $18,2 \times 10^{-3}$, e produziu uma taxa de erro de 0, 0536. Cabe notar que este receptor novamente teve o melhor desempenho de MSWE e a menor taxa de erro de símbolo, dentre os esquemas baseados em algoritmos LMS de passo variável.

Também foram levantadas curvas de MSWE com RSR de $20 \mathrm{~dB}$ e demais condições idênticas às da Figura 10. Verificou mais uma vez que o esquema baseado no algoritmo MVSSLMS apresentou alta velocidade de convergência e atingiu o menor nível de MSWE em regime permanente, dentre os receptores baseados em algoritmos LMS.

Em suma, em todas as simulações realizadas, o desempenho obtido com o algoritmo MVSS-LMS só foi superado pelo produzido com o RLS, tanto no que se refere à velocidade de convergência e ao nível de MSWE em regime permanente, quanto à taxa de erros de símbolos.

Neste ponto, cabe notar que a complexidade computacional do algoritmo MVSS-LMS é muito menor que a do RLS, uma vez que ele requer apenas o cálculo de uma seqüência de valores do passo utilizando parâmetros do sistema de transmissão e do canal. Como o MVSS-LMS se mostrou bastante robusto a erros de estimação destes parâmetros, podem ser utilizados estimadores de baixa complexidade computational para cálculo de suas estimativas, além de não haver necessidade de atualizálas na velocidade com que eventualmente variem.

Considerando ainda que não se empregam amostras do sinal recebido para o cálculo da sequiência de passos do MVSSLMS, vê-se que há ainda possibilidades interessantes para exploração de períodos de ociosidade dos processadores, no caso de implementações em tempo real deste algoritmo.

\section{CONClusões}

Neste artigo foi feita uma comparação de desempenho de receptores MLSE/PSP baseados no emprego de diferentes algoritmos de filtragem adaptativa para estimação de canal, tendo como foco principal alguns algoritmos LMS de passo adaptativo recentemente propostos.

Utilizou-se como indicadores de desempenho a taxa de erros de símbolo do receptor e o erro médio quadrático na estimação dos coeficientes da resposta ao impulso do canal. Estes parâmetros foram estimados a partir da simulação em computador de diversos cenários de transmissão, caracterizados pela razão sinal-ruído na entrada do receptor e pelo desvio Doppler máximo. Também se investigou a robustez de desempenho dos algoritmos LMS de passo variável diante de variações de parâmetros empregados em suas regras de adaptação do passo.

Verificou-se que o algoritmo MVSS-LMS apresentou características de desempenho muito superiores às dos outros algoritmos LMS aqui considerados, sendo suplantado apenas pelo algoritmo RLS. Resultados apresentados neste trabalho também mostraram que o desempenho do MVSS-LMS é bastante robusto a erros na estimação de parâmetros por ele empregados na variação do passo.

Considerando-se estas características, e o fato de que o MVSS-LMS tem complexidade muito inferior à do RLS, podese afirmar que se trata de uma ferramenta potencialmente útil para viabilizar aplicações de receptores adaptativos MLSEPSP, em diversos sistemas e cenários de comunicações atualmente sob investigação.

\section{REFERÊNCIAS}

[1] R. Raheli, A. Polydoros, and C.Tzou, "Per-survivor processing: A general approach to MLSE in uncertain environments," IEEE Transactions on Communications, vol. 43, no. 2/3/4, pp. 354-364, February/March/April 1995.

[2] J. F. Galdino and E. L. Pinto, "A simulation study of adaptive filtering applied to MLSE-PSP receivers," in IEEE Military Communications Conference (MILCOM), 1998.

[3] Z. Zhu and H. R. Sadjadpour, "An adaptive per-survivor processing algorithm," IEEE Transactions on Communications, vol. 50, pp. 17161718, November 2002.

[4] Z. Li and A. Mämmelä, "An adaptive RSSE-PSP receiver with a prefilter for EDGE systems," in 2003 IEEE International Conference on Communications (ICC'03), vol. 5, 2003, pp. $1252-1256$.

[5] C. Cozzo and B. L. Hughes, "An adaptive receiver for space-time trellis codes based on per-survivor processing," IEEE Transactions on Communications, vol. 50, pp. 1213-1216, August 2002.

[6] Y. Xue and X. Zhu, "Per-survivor processing-based decoding for spacetime trellis code," IEEE Transactions on Vehicular Technology, vol. 52, pp. 1173-1178, July 2003.

[7] M. E. Rollins and S. J. Simmons, "Simplified per-survivor kalman processing in fast frequency-selective fading channels," IEEE Transactions on Communications, vol. 45, no. 5, pp. 544-553, May 1997.

[8] C. D. Harris, R.W. and F. Bishop, "A variable step (VS) adaptive filter algorithm," ASSP-34 - IEEE Trans., pp. 309-316, 1986.

[9] R. H. Kwong and E. W. Johnston, "A variable step size LMS algorithm," IEEE Transactions on Signal Processing, vol. 40, no. 7, pp. 1633-1642, July 1992.

[10] G. Keratiotis and L. Lind, "Optimum variable step-size sequence for LMS adaptive filters," IEE Proceedings Vis. Image Signal Process., vol. 146, no. 1, pp. 1-6, February 1999.

[11] Y. Gu, K. Tang, H. Cui, and W. Du, "Optimal step-size update equation in nonstationary environment and OVS-LMSII algorithm," in IEEE 2002 International Conference on Communications, Circuits and Systems and West Sino Expositions, 2002, pp. 1252 - 1256.

[12] A. M. Arraes, E. L. Pinto, and J. F. Galdino, "A new variable stepsize LMS algorithm for the estimation of time-varying and frequencyselective channels," Electron. Lett., IEE, vol. 40, no. 20, pp. 1312-1313, September 2004.

[13] J. D. Parsons, The Mobile Radio Propagation Channel. John Wiley, 1992.

[14] J. G. Proakis, Digital Communications. McGraw-Hill, 1995.

[15] G. D. J. Forney, "The viterbi algorithm," Proceedings of the IEEE, vol. 61, no. 3, pp. 268-278, March 1973.

[16] J. B. Anderson and S. Mohan, "Sequential coding algorithms: a survey and cost analysis," IEEE Transactions on Communications, vol. COM32, pp. 169-176, February 1984.

[17] A. M. Arraes, Algoritmos LMS de Passo Variável na Identificação de Canais WSS-US. Dissertação de Mestrado, IME, 2005.

[18] A. Feuer and E. Weinstein, "Convergence analysis of LMS filters with uncorrelated gaussian data," In: IEE Trans. Acoust., Speech, Signal Processing, vol. ASSP-34, pp. 222-230, February 1985.

[19] A. M. Arraes, E. L. Pinto, and J. F. Galdino, "A variable step-size LMS algorithm for channel estimation based on MSWE minimization," Artigo submetido ao IET Transactions on Communications, 2007.

[20] J. F. Galdino, E. L. Pinto, and M. S. Alencar, "Analytical performance of the LMS algorithm on the estimation of wide sense stationary channels," IEEE Transactions on Communications, vol. 52, no. 6, pp. 982-993, Jun 2004. 